alongside an expectation from funding bodies to go beyond publication within academic journals has generated interest in alternative methods of science communication.

Our aim is to share and describe our experience of using a variety of creative communication tools, reflect on their use in different situations, enhance learning and generate discussion within the research community.

Methods Over the last 4 years, we have explored a number of creative communication tools (illustration, podcasts, blogs, briefing papers, board games and social media shareable content) to share our research with the diverse range of people who could benefit from the findings and extend dissemination beyond traditional academic mechanisms.

Central to our approach is the co-production of a communication plan with potential evidence users which facilitates i) the identification of key messages for different audiences, ii) discussion of appropriate tools to communicate the key messages and iii) exploration of avenues to share them. We aim to involve evidence users in the production of tools where appropriate and to produce a variety of outputs for each research project cognisant of the many ways in which individuals choose to engage with information.

Results Our experience has allowed us to develop an understanding of the pros and cons of a wide range of creative communication tools. For example, board games can be a fun way of learning, may flatten power hierarchies between researchers and research users and enable the sharing of large amounts of complex information in a thought provoking way but they are time and resource intensive both to produce and to engage with. Conversely, social media shareable content can be quick and easy to produce and to engage with but limited in the depth and complexity of shareable information. This method also requires careful consideration of the social media use and habits of the target audience.

Based on this experience, we have developed a simple infographic to guide future decision making.

Conclusion It is widely acknowledged that most stakeholders do not have time to invest in reading large and complex documents; creative communication tools can be a useful mechanism to improve accessibility to key messages. Furthermore, our experience has highlighted a range of additional benefits of embedding these techniques within our project management processes e.g. opening up two-way conversation with end-users of research to discuss the implications of findings.

\section{P55 RACIAL DISCRIMINATION PREDICTS BODY WEIGHT GAIN IN BLACK INDIVIDUALS IN FOUR YEARS FOLLOW-UP OF THE ELSA-BRASIL COHORT}

\begin{abstract}
${ }^{1,2} \mathrm{SM}$ Barreto*,${ }^{2} \mathrm{AV}$ Machado, ${ }^{2} \mathrm{ST}$ Menezes, ${ }^{3} \mathrm{D}$ Chor, ${ }^{4} \mathrm{RH}$ Griep, ${ }^{3} \mathrm{JMN}$ Guimarães, ${ }^{5}$ SMA Matos, ${ }^{1,2}$ L Giatti, ${ }^{1,2}$ LV Camelo. 'Departament of Preventive Medicine, Universidade Federal de Minas Gerais, Belo Horizonte, Brazil; ${ }^{2}$ Post Graduate Program of Public Health, Universidade Federal de Minas Gerais, Belo Horizonte, Brazil; ${ }^{3}$ National School of Public Health, Fundação Oswaldo Cruz, Rio de Janeiro, Brazil; ${ }^{4}$ Laboratory of Health and Environment Education, Fundação Oswaldo Cruz, Rio de Janeiro, Brazil; ${ }^{5}$ Institute of Collective Health, Universidade Federal da Bahia, Salvador, Brazil
\end{abstract}

\subsection{6/jech-2020-SSMabstracts. 148}

Background Obesity is a worldwide public health problem, and large disparities between racial groups have been reported. The North American literature suggests that racial discrimination can accelerate the increase in body weight throughout life. As racism in Brazil has different and specific facets, our study aims to describe the racial difference in body weight and BMI $\left(\mathrm{kg} / \mathrm{m}^{2}\right)$ gain in four-years follow-up and to investigate whether racial discrimination changes these trajectories among Black and Brown individuals.

Methods The present study compares body weight and BMI changes between the 1st (2008-2010) and the 2nd visits (2012-2014) of 13,133 participants of the Brazilian Longitudinal Study of Adult Health (ELSA-Brasil) according to race/skin color. In order to investigate whether racial discrimination predicts greater weight and BMI gain in Blacks and Browns $(\mathrm{N}=5,983)$, we used mixed-regression models. Information on racial discrimination, assessed by the modified version of the Lifetime Major Events Scale, and on sociodemographic, behavioral, and depressive episode factors were obtained at the 1 st visit.

Results Mean age of participants was 52 years, 54\% were female, $54 \%$ self-declared as White, $29 \%$ as Brown and $17 \%$ as Black. The median follow-up time was 3.9 years. During the study period Whites, Blacks and Browns gained an average $1.2 \mathrm{~kg}, 1.2 \mathrm{~kg}$, and $1.4 \mathrm{~kg}$, respectively, corresponding to an average increase in BMI of $0.60,0.56$ and $0.65 \mathrm{~kg} / \mathrm{m}^{2}$, respectively $(\mathrm{p}>0.05)$. Racial was reported by $6.3 \%$ of Brown and $32.1 \%$ of Black participants. The weight gain was greater among Black $(2.1 \mathrm{~kg}$ versus $1.0 \mathrm{~kg}$, $\mathrm{p}<0.001)$ and Brown individuals $(1.9 \mathrm{~kg}$ versus $1.1 \mathrm{~kg}$, $\mathrm{p}=0,02$ ) who reported racial discrimination as compared to those who did not report. Mixed-effects regression models demonstrated that Blacks who reported racial discrimination had greater weight and BMI increases than those who did not report, even after adjustment by potential confounders (age, sex, education and income). These results remained statistically significant even after adjusting for mediating variables of the association between discrimination and weight/ BMI gain (smoking, physical activity, alcohol consumption and depressive episode). In Browns, racial discrimination was not an independent predictor of weight gain and BMI over time.

Conclusion Weight and BMI gains were observed among all race/skin color groups examined, with no statistical differences between them. The results indicate that Black individuals who reported racial discrimination had greater weight and BMI increases in the study period than those who did not, reinforcing the importance of public policies against racial discrimination to reduce racial disparities in health.

\section{P56 PROMOTING HEALTHY INFANT FEEDING TO PREVENT CHILDHOOD OBESITY: PROCESS EVALUATION OF THE CHOOSING HEALTHY EATING FOR INFANT HEALTH (CHERISH) INTERVENTION}

${ }^{1} \mathrm{C}$ Flannery*, ${ }^{1} \mathrm{~K}$ O'Neill, ${ }^{1} \mathrm{~S}$ Calnan, ${ }^{1} \mathrm{H}$ McGrath, ${ }^{1} \mathrm{~K}$ Matvienko-Sikar, ${ }^{2} \mathrm{E}$ Toomey, ${ }^{2} \mathrm{M}$ Byrne, ${ }^{1} \mathrm{PM}$ Kearney, on behalf of the CHErlsH research team. ' $\mathrm{Sch}$ ool of Public Health, University College Cork, Cork, Ireland; ${ }^{2}$ Health Behaviour Change Research Group, School of Psychology, National University of Ireland, Galway, Ireland

\subsection{6/jech-2020-SSMabstracts. 149}

Background The CHErIsH Choosing Healthy Eating for Infant Health intervention is a brief intervention to be delivered during routine vaccination visits, to improve healthy infant feeding behaviours among parents to help reduce the risk of childhood obesity. An accompanying implementation strategy 
was designed to support delivery of CHErIsH by healthcare practitioners (HCPs) delivering the intervention. This strategy included appointing a local opinion leader and providing two incentivised training sessions and educational materials. The aim of this study was to examine the acceptability and feasibility of this HCP-level implementation strategy.

Methods A mixed-methods approach using qualitative and quantitative methods was used to investigate the acceptability and feasibility of the HCP-level implementation strategy. HCPs were recruited from three practices within a Healthcare Centre involved in the implementation of the intervention. Data were collected using researcher observations and field notes; HCP checklists, questionnaires and focus groups with Practice Nurses (PNs) and General Practitioners (GPs). Thematic analysis of qualitative data and statistical analysis of quantitative data is on-going.

Results Of the 21 HCPs involved in the implementation strategy, $18(85.7 \%)$ completed both the pre and post-training questionnaires (GPs=9; PNs=9). Prior to training, $88 \%$ of HCPs considered discussing infant feeding as part of their professional role; however, only $44 \%$ reported feeling confident in doing so, with no HCPs discussing infant feeding at 4 and 6 months. The most common source of infant feeding information reported by HCPs included health service leaflets, discussions with colleagues and information leaflets from infant formula manufacturers. After training, all HCPs considered infant feeding to be part of their professional role, with $31 \%$ now discussing infant feeding with parents for infants aged 4 and 6 months. Qualitative data indicate that HCPs felt that both training sessions which provided resources and educational materials, were 'useful and very productive'.

Conclusion CHErIsH training sessions delivered as part of the implementation strategy were considered acceptable and feasible by the HCPs, and improved their confidence in providing infant feeding advice. These training sessions, which include information on current national feeding guidelines and feeding issues, should be delivered as part of routine HCP training. This will ensure that all HCPs have evidence-based training to support the delivery of consistent infant feeding messages at infant vaccination visits in primary care practice.

\section{\begin{tabular}{|l|l}
\hline P57 CUTS TO SPENDING ON SURE START CHILDREN'S \\
\hline
\end{tabular} CENTRES AND CHILDHOOD OBESITY: EVIDENCE FROM A NATIONAL, LONGITUDINAL ECOLOGICAL STUDY IN ENGLAND}

KE Mason*, A Alexiou, D Bennett, B Barr, D Taylor-Robinson. Public Health and Policy, University of Liverpool, Liverpool, UK

\subsection{6/jech-2020-SSMabstracts. 150}

Background Childhood obesity is rising in disadvantaged areas in the UK. Whilst the causes of childhood obesity are multiple and complex, modifiable pathways may operate through provision of community-based services in the early years, where healthy nutrition and physical activity can be supported in numerous direct and indirect ways. In England, local authority-run Sure Start children's centres have been an important source of parenting programmes; early learning and childcare; promotion of breastfeeding, active play and good nutrition; and links with employment and welfare support.

Austerity measures adopted by the UK government since 2010 resulted in large cuts to local authority (LA) budgets and, consequently, dramatic reductions in many councils' expenditure on non-statutory services, including Sure Start centres. Reduced investment in these centres may affect a range of child health and development outcomes, including childhood obesity. We assessed whether spending cuts were associated with the prevalence of obesity at school reception (age 4-5 years).

Methods This is a longitudinal ecological study over the period 2010-2017. Our main exposure was LA expenditure on Sure Start centres using data from the Department for Education. Our outcome was obesity prevalence at reception, using data from the National Child Measurement Programme. We used fixed-effects panel regression, controlling for secular changes in obesity prevalence and time-varying confounding by local economic conditions and levels of child poverty. We examined interactions with LA deprivation and pre-2010 obesity trends, and conducted a negative control analysis using spending on services for older children as a control exposure. Results Between 2010 and 2017, spending on Sure Start and early years' services decreased by $56 \%$ on average across LAs in England, and more in deprived areas. Childhood obesity prevalence plateaued overall, but continued to increase in some areas, particularly more deprived areas. Our analysis indicates that, on average, obesity prevalence increased in areas with larger cuts to Sure Start spending. We estimate that each $10 \%$ spending cut is associated with a $0.34 \%$ relative increase in obesity prevalence (95\% CI:0.15\%-0.53\%). There is some evidence that this association is stronger in the most deprived areas and in areas where obesity prevalence had been falling prior to 2010 .

Conclusion Our findings suggest cuts to local authority spending on children's centres are associated with increased childhood obesity. Disinvestment in the services these centres provide in the early years may be undermining progress in reducing the prevalence of childhood obesity. Limitations of the study include a reliance on area-level obesity data.

\section{P58 COMBINED EFFECTS OF SOCIAL RELATIONSHIPS AND VISCERAL ADIPOSITY IN WOMEN AND MEN}

${ }^{1,2} \mathrm{Al}$ Conklin*, ${ }^{1} \mathrm{Z}$ Hosseini, ${ }^{3} \mathrm{G}$ Veenstra, ${ }^{2,4} \mathrm{NA}$ Khan. ${ }^{1}$ Faculty of Pharmaceutical Sciences, University of British Columbia, Vancouver, Canada; ${ }^{2}$ Centre for Health Evaluation and Outcome Sciences, Providence Health Research Institute, Vancouver, Canada; ${ }^{3}$ Department of Sociology, University of British Columbia, Vancouver, Canada; ${ }^{4}$ Faculty of Medicine, University of British Columbia, Vancouver, Canada

\subsection{6/jech-2020-SSMabstracts. 151}

Background Social connections are recognized as a major determinant of survival and wellbeing. Around half of Canadians aged 80 and older live alone and more senior women live alone than senior men. The link between different social ties and central obesity in women and men is poorly understood, and the interplay of different social ties is seldom studied. This population-based study examined multiple structural social ties in relation to waist circumference (WC) among women and men in Canada.

Methods We used baseline data from the population-based Canadian Longitudinal Study on Aging (2012-2015) Comprehensive cohort of 28,238 adults (45-85 years), and genderstratified multivariable linear regression models with interaction terms for each pair of social ties. Regression coefficients were used in post-estimation calculation of adjusted means and $95 \%$ confidence intervals. 